\title{
Unprovoked hemothorax: An unusual presentation in Dengue Haemorrhagic Fever
}

\author{
Robin George Manappallil \\ Consultant- Physician, Department of Medicine, National Hospital, Calicut, Kerala, India
}

According to WHO, Dengue fever (DF) is the most rapidly spreading mosquito-borne viral disease in the world. Dengue Haemorrhagic fever (DHF) is a severe and more serious form of DF, characterised by fever, bleeding manifestations, plasma leakage and thrombocytopenia. This is a case of a 28 year old male, who presented with history of fever and myalgia and was diagnosed to have DF. He had developed acute onset dyspnoea and his chest X-Ray showed right sided pleural effusion. Pleurocentesis revealed haemorrhagic fluid in the absence of trauma. Unprovoked hemothorax as an initial presentation of DHF has rarely been reported.

\section{Access this article online}

Website:

http://nepjol.info/index.php/AJMS DOI: 10.3126/ajms.v7i3.13816 E-ISSN: 2091-0576 P-ISSN: $2467-9100$

Key words: Hemothorax, Dengue hemorrhagic fever, Disseminated intravascular coagulation

\section{INTRODUCTION}

Dengue virus infection may be asymptomatic. It may also present as an undifferentiated fever, which is a simple fever indistinguishable from other viral infections. According to WHO, dengue fever (DF) is an acute febrile illness of 2-7 days duration with two or more of the following: headache, retro-orbital pain, myalgia/arthralgia, rash, haemorrhagic manifestation (petechiae, positive tourniquet test), leucopenia. The complications of DF, though infrequent, are usually associated with the more severe forms i.e. hemorrhagic and shock syndrome. The most serious complications include dehydration, hemorrhage, hypotension, bradycardia, seizures, encephalitis and death. ${ }^{1}$

The patient being reported is a case of unilateral unprovoked hemothorax presenting as an initial bleeding manifestation of DHF, which is a rare presentation.

\section{CASE REPORT}

The patient is a 28 year old male, a tailor, who presented to emergency department with 3 days history high grade fever and myalgia. On presentation, he was febrile with temperature of $101^{\circ} \mathrm{F}$ while his other vitals were stable.
His saturation was $98 \%$ in room air. He had diffused erythematous rash. His systemic examinations were normal. His blood investigations showed hemoconcentration with $\mathrm{Hb}$ of $17 \mathrm{~g} / \mathrm{dL}(13-16 \mathrm{~g} / \mathrm{dL})$ and PCV 50\% (39 - 46 \%), leucopenia of 3,200/cumm (4000 - 10000/cumm) and thrombocytopenia of 20,000/cumm (1.5 - 4.5 lakhs/cumm). His liver enzymes were elevated i.e. SGOT $152 \mathrm{U} / \mathrm{L}$ (12 - $38 \mathrm{U} / \mathrm{L})$ and SGPT $324 \mathrm{U} / \mathrm{L}(7-41 \mathrm{U} / \mathrm{L})$, but renal functions were normal. His activated thromboplastin time was slightly prolonged (test 49 seconds, control 24 seconds). Dengue NS1 antigen was positive. His chest X-Ray and ECG were normal. He had a positive tourniquet test. He was treated symptomatically with intravenous fluids.

On day 2, patient became afebrile but his platelet count remained unchanged. He did not have any bleeding manifestations, except 20 - 25 RBCs in urine microscopy. He complained of mild abdominal discomfort. Ryles tube was placed and gastric aspirate contained only food particles. His amylase and lipase levels were within normal reference range. Abdominal examination showed shifting dullness and ultrasound abdomen revealed moderate ascites. His breath sounds were decreased over right infra-axillary and infrascapular regions with stony dull note on percussion, and chest $\mathrm{X}$-Ray showed mild right sided pleural effusion. He was given prophylactic platelet transfusion in view of mild hematuria. 
On day 3 , he started becoming tachypnoeic but saturation was $94 \%$ in room air. His chest X-Ray showed an increase in right sided pleural effusion. By day 4, his tachypnoea increased with saturation dropping between $85-88 \%$. His chest X-Ray showed further increase in pleural effusion. During these two days, his platelet count remained between 22,000 and $28,000 /$ cumm.

On day 5, because of his increasing tachypnoea, platelet and fresh frozen plasma (FFP) transfusions were given and therapeutic pleural tap was done, which revealed haemorrhagic fluid. The pleural fluid to serum PCV was suggestive of hemothorax. An intercostal drain (ICD) was inserted and about $800 \mathrm{ml}$ of haemorrhagic fluid was drained, following which patient became symptomatically better. He was given whole blood, platelet and FFP transfusions.

By day 7, patient developed hematuria and hematochezia. His blood investigations showed $\mathrm{Hb}$ of $10 \mathrm{~g} / \mathrm{dL}$, platelet of $32,000 /$ cumm, INR of 4.2, prolonged aptt (test 68 seconds, control 28 seconds) and positive D-dimer, suggestive of DIC. He had a continuous flow of blood from the ICD. He went into severe hypotension and bradycardia. Resuscitative measures were started but patient could not be revived and expired on same day.

\section{DISCUSSION}

The Dengue virus is a small single stranded RNA virus comprising of four distinct serotypes (DENV 1-4). Primary infection provides lifelong immunity against that infecting serotype, but there is no long-term cross protective immunity against other serotypes. ${ }^{2}$

Severe dengue is characterized by plasma leakage, haemoconcentration and abnormal homeostasis. ${ }^{1}$ Plasma leakage is mediated via endothelial cell activation and histamine release. ${ }^{3,4}$ The infection of human haematopoietic cells causes alterations in megakaryocytopoieses, leading to thrombocytopenia. Haemorrhage can occur as a result of thrombocytopenia or DIC. ${ }^{1}$ Severe dengue associated with secondary infection may be due to antibody-dependent enhancement of the infection. ${ }^{5}$

There are three phases in DF. The first phase is called febrile phase and is characterized by fever, headache, myalgia and leucopenia. It lasts for 2-7 days. The second phase is the critical phase, where there is associated thrombocytopenia and increase in haematocrit level. There is a drop in temperature and increase in capillary permeability. Pleural effusion and ascites may be detectable. Severe plasma leakage may lead to shock. There may also be organ involvement in the form of hepatitis, myocarditis, encephalitis or severe bleeding. This phase lasts for 24-48 hours. The last is the recovery phase where there is gradual resorption of extracellular compartment fluid. The platelet count rises and haematocrit start stabilizing. Respiratory distress may occur due to massive pleural effusion and ascites, if there was excessive fluid resuscitation. ${ }^{1}$

Pleural effusion is a feature of severe dengue due to plasma leakage. Other respiratory conditions like pneumonitis, acute respiratory distress syndrome and pulmonary haemorrhage are rare. There have been reports of cases with bloody pleural effusion. ${ }^{6-8}$ However, in this case, the patient presented at the end of febrile phase. And as he went through his critical phase he developed pleural effusion which was found to be hemothorax. Though he attained symptomatic relief following ICD insertion, he fell prey to the complication of DIC and expired.

This case emphasises the need to consider hemothorax when a patient with DF develops pleural effusion. This is a rather rare presentation and warrants aggressive supportive therapy and transfusions, in the form of whole blood, platelets and FFP, in order to maintain hemodynamic stability.

\section{REFERENCES}

1. WHO. Dengue: guidelines for diagnosis, treatment, prevention and control. New edition. Geneva: World Health Organization; 2009.

2. Halstead SB. Etiologies of the experimental dengues of Siler and Simmons. American Journal of Tropical Medicine and Hygiene 1974; 23:974-982.

3. Avirutnan $P$, Malasit $P$, Seliger B, Bhakdi S and Husmann M. Dengue virus infection of human endothelial cells leads to chemokine production, complement activation, and apoptosis. Journal of Immunology 1998; 161:6338-6346.

4. Setlik RF, Ouellette D, Morgan J, McAllister CK, Dorsey D, Agan BK, et al. Pulmonary haemorrhage syndrome associated with an autochthonous case of dengue haemorrhagic fever. Southern Med J 2004; 97: 688-691.

5. Halstead SB. Antibody, macrophages, dengue virus infection, shock, and hemorrhage: a pathogenetic cascade. Reviews of Infectious Diseases 1989; 11(Suppl 4):S830-S839.

6. Wang CC, Liu SF, Liao SC, Lee IK, Liu JW, Lin AS, et al. Acute respiratory failure in adult patients with dengue virus infection. Am J Trop Med Hyg 2007; 77: 151-158.

7. Karanth SS, Gupta A and Prabhu M. Unilateral massive hemothorax in Dengue hemorrhagic fever: a unique presentation. Asian Pac J Trop Med 2012; 5(9):753-754.

8. Anam AM, Rabbani R, Shumy F and Polash MM. Subsequent pancreatitis and haemothorax in a patient of expanded dengue syndrome. Trop Doct 2015; 27. 